\title{
Suppression of thermosensitive peptidyl-tRNA hydrolase mutation in Escherichia coli by gene duplication
}

\author{
Jeanne Menez ${ }^{1}$, Elisabeth Remy ${ }^{2}$ and Richard H. Buckingham ${ }^{1}$ \\ Author for correspondence: Richard H. Buckingham. Tel: +33 158415120 . Fax: +33158415020. \\ e-mail: rhb@ibpc.fr
}

1 UPR9073 du CNRS, Institut de Biologie Physico-

Chimique, 13 rue Pierre et Marie Curie, F-75005 Paris, France

2 Aventis Pharma SA, 13 Quai Jules Guesde, 94403 Vitry sur Seine Cedex, France

\begin{abstract}
Peptidyl-tRNA hydrolase (Pth) in Escherichia coli is required to recycle tRNA molecules that dissociate from the ribosome as peptidyl-tRNA during protein synthesis. At non-permissive temperatures, strains with a thermosensitive mutation affecting the enzyme accumulate peptidyl-tRNA, cease protein synthesis and die. The rate of reversion of this mutation to thermoresistance varies widely according to the genetic background of the cell and the temperature of selection; under certain conditions, reversion can occur at rates approaching $10^{-3}$ per cell per generation. In such revertants, a chromosomal pth gene can be replaced by an inactivated gene, restoring thermosensitive growth in most cases. PCR amplification experiments and Southern blots show the presence of both normal and inactivated copies of the gene, demonstrating that gene duplication has occurred in the revertants. Estimation of intracellular peptidyl-tRNA hydrolase by Western blotting confirms this explanation of the mechanism of high-frequency reversion to thermoresistance.
\end{abstract}

Keywords: translation, ribosome, termination

\section{INTRODUCTION}

During normal protein synthesis, the ribosome forms initiation complexes on mRNA, performs repeated cycles of elongation as the mRNA is decoded, and releases the polypeptide chain by hydrolysis of peptidyltRNA when it encounters a termination signal on mRNA. However, protein synthesis is not perfectly processive, and peptidyl-tRNA dissociates prematurely from the ribosome at a frequency thought to be about $3 \times 10^{-4}$ per codon (Jørgensen \& Kurland, 1990; Manley, 1978). Peptidyl-tRNA released in the cell is hydrolysed by peptidyl-tRNA hydrolase (Pth), an enzyme that appears to be ubiquitous and has been shown to be essential in Escherichia coli. Pth is an esterase, able to recycle $\mathrm{N}$-acetyl-aminoacyl-tRNA and peptidyltRNA by cleaving the ester bond between tRNA and peptide (Vogel et al., 1968). In the absence of Pth activity, accumulation of some peptidyl-tRNA species in the cell is rapid, and protein synthesis becomes inhibited due to lack of aminoacyl-tRNA, leading to cell death. Certain tRNAs accumulate as peptidyl-tRNA much faster than others, and starvation in E. coli has been found to occur first for tRNA ${ }^{\mathrm{Lys}}$ (Heurgué-Hamard et al., 1996; Menninger, 1978).

Studies of premature peptidyl-tRNA dissociation (or drop-off) have been greatly facilitated as a result of the isolation of a conditional lethal temperature-sensitive mutant affecting pth (Atherly \& Menninger, 1972). Bacteria harbouring this mutation stop growing after a shift to non-permissive temperatures, following the accumulation of peptidyl-tRNA and the inhibition of protein synthesis. The mutation was identified as a single nucleotide change leading to an amino-acid substitution Gly100Asp (De La Vega et al., 1996). Though distant from the active site proposed in the three-dimensional structure (Schmitt et al., 1997), this Gly residue is highly conserved in Pth from many different organisms. Recent work has shown that the mutation leads to a Pth protein that is unstable in vivo, at both permissive and non-permissive temperatures, but which shows a specific activity comparable to that of the wild-type enzyme (Cruz-Vera et al., 2000). The mutant enzyme seems not to be correctly folded and is subject to degradation by ClpP and Lon proteases (CruzVera et al., 2000).

In addition to allowing studies of peptidyl-tRNA accumulation, the thermosensitive Pth mutant has led to improved understanding of the drop-off process as a result of the isolation and characterization of extragenic revertants and multicopy suppressors of the $p t h(\mathrm{ts})$ mutation. Thus, overproduction of tRNA ${ }^{\text {Lys }}$ raises 
considerably the threshold for thermosensitive growth by promoting more synthesis of the tRNA for which starvation occurs first (Heurgué-Hamard et al., 1996). GroESL overproduction, presumably by improving the efficiency of mutant Pth protein folding, increases Pth activity. Apart from revertants directly affecting the $p t h$ gene, mutations inactivating termination factor RF3, or reducing the intracellular concentration of ribosome recycling factor RRF, suppress the $p t h(t s)$ mutation. In vitro experiments show that suppression is a result of a reduced frequency of peptidyl-tRNA drop-off in the cell (Heurgué-Hamard et al., 1998). The studies that led to the isolation of these and other suppressors of $p t h(t s)$ showed that under some conditions of selection and in some strain backgrounds high rates of reversion to thermoresistance were observed. Here we examine this finding in more detail and show that the high rates of reversion are due to duplication of the $p t h$ (ts) gene.

\section{METHODS}

Bacterial strains and plasmids. Escherichia coli K-12 strains are listed in Table 1. FTP4993 and C600 were used as wildtype strains. JMM35 was constructed as follows. First, the kanamycin-resistance gene $(\mathrm{Kn})$ from $\mathrm{Tn} 903$, cloned by flanking BamHI sites (GenBank J01839, bp 697-2392) into the BamHI site of the polylinker in BlueScribe $\mathrm{M} 13+$ (Stratagene), was excised by digestion with HincII and EcoRI and blunted by Klenow PolA. This fragment was cloned into the HincII site of the $p t h(\mathrm{ts})$ gene carried in plasmid pVH227 (a pWSK129 derivative, V. Heurgué-Hamard, UPR9073, CNRS, Paris, unpublished results), interrupting the Pth coding sequence after 28 amino acids. The KpnI-Pst I fragment carrying the interrupted $p t h$ gene from this construction was then cloned into plasmid pMAK705, which is thermosensitive for replication (Hamilton et al., 1989), giving plasmid pMAKpth::Kn. This plasmid allows recombination of $p t h:: \mathrm{Kn}$ at the $p t h$ chromosomal locus. Recombination and resolution of the integrated plasmid was performed as described by Blomfield et al. (1991) to obtain strain VH1004, which harbours $p t h:: \mathrm{Kn}$ in the chromosome of strain FTP4993 and a plasmid encoding wild-type $p t h$, pMAKpth. A further plasmid carrying wild-type $p t h$ (pBADpth) was constructed by amplifying the $p t h$ gene with primers introducing EcoRI and HindIII sites at the beginning and end of the coding sequence. These sites were then used to clone the fragment containing pth between the same sites in plasmid pBAD30 (Guzman et al., 1995) cut by the same enzymes, placing the $p t h$ gene under the control of an arabinose-inducible promoter. Strain JMM35 was then constructed by P1 transduction of the $p t h::$ Kn locus from VH1004 to strain FTP4933 transformed with plasmid pBADpth in the presence of $0.2 \%$ arabinose. This strain depends on the presence of arabinose for normal growth.

Recombinant DNA techniques and genetic manipulations. General procedures for recombinant DNA techniques, plasmid extraction, agarose gel electrophoresis, etc., were performed as described by Sambrook et al. (1989). DNA fragments from agarose gels were extracted using Jetsorb gel (Bioprobe) according to the manufacturer's instructions. Phage P1 lysates, transductions and transformations were performed as described by Miller (1992).

Growth conditions. Luria-Bertani broth (LB) was employed as rich medium. Antibiotics were added as necessary at the following final concentrations: $50 \mu \mathrm{g}$ kanamycin $\mathrm{ml}^{-1}, 200 \mu \mathrm{g}$ ampicillin $\mathrm{ml}^{-1}$. Growth experiments at different temperatures were performed on agar plates. For pBAD30-derived plasmids, arabinose was added for induction at $0.02 \%$ for expression of $p t h$ at about chromosomal level or at $0 \cdot 2 \%$ for maximum promoter activity.

Determination of reversion rates. Mutation rates were measured as described by Luria \& Delbrück (1943). Serial dilutions (typically from $10^{-7}$ to $10^{-12}$ ) of an overnight culture of the $p t h$ (ts) mutant strains were made and grown overnight at $30^{\circ} \mathrm{C}$. The last dilution that grew was used to prepare 30 independent cultures each containing 20-50 bacterial cells in $200 \mu \mathrm{l}$ rich medium. These were grown separately at permissive temperature $\left(30^{\circ} \mathrm{C}\right)$ for several hours, the period being determined by preliminary experiments such that about half the cultures acquired no revertants. Each culture was then completely plated on preheated agar plates and grown at nonpermissive temperature. The number of plates on which no clones appeared allows determination of the mutation rate according to the equation $P=-(1 / N) \ln F$, where $P$ is the mutation rate per cell per generation, $N$ the total number of bacterial cells per culture, and $F$ the fraction of cultures acquiring no revertants (Luria \& Delbrück, 1943).

PCR amplification of pth and pth::Kn. The presence of the intact and interrupted $p t h$ gene was verified by PCR using the pairs of oligonucleotide primers pt1-pt5, pt1-mk6 and mk2-

\section{Table 1. Bacterial strains}

\begin{tabular}{|c|c|c|c|}
\hline Strain & Genotype & Derivation/use & Source \\
\hline $\mathrm{Xac}$ & $\Delta($ lac-pro $)$ argE ara gyrA rpoB thi & pth wild-type & Coulondre \& Miller (1977) \\
\hline FTP4993 & metB glyV55 trpA(AGC233, UGA234) & $p t h$ wild-type & $\begin{array}{l}\text { F. T. Pagel, University of } \\
\text { Texas }\end{array}$ \\
\hline VH733 & metB glyV55 pth(ts) & FTP4993 by several transductions & Heurgué-Hamard et al. (1998) \\
\hline C600 & thr-1 leu-6 thi-1 supE44 tonA lacY1 & $p t h$ wild-type & $\begin{array}{l}\text { G. Guarneros, CINVESTAN- } \\
\text { IPN, Mexico City }\end{array}$ \\
\hline VH4 & $\mathrm{C} 600, p t h(\mathrm{ts})$ & C600 derivative & Heurgué-Hamard et al. (1996) \\
\hline VH805 & metB glyV55 pth(ts) recA:: Cm & $\begin{array}{l}\text { VH733 by transduction of } \\
\operatorname{rec} A:: \mathrm{Cm}\end{array}$ & Menez et al. (2000) \\
\hline VH1004 & FTP4993, pth:: Kn, pMAKpth & & This work \\
\hline JMM35 & FTP4993, pth:: Kn, pBADpth & & This work \\
\hline JMM101 & FTP4993, pth::Kn, pBADpth(ts) & & This work \\
\hline
\end{tabular}


pt5 shown in Fig. 1. Primer sequences were: mk2, TGATGTTACAGATGAGATGGTC; mk6, CGCCTGAGCGAGACGA; pt1, GAATTCAATGGCACCGACGAAAATAC; pt5, GGGACTAACAGGCGGACA.

Western blotting. Western blot experiments were performed using rabbit anti-Pth antibodies commercially prepared from pure Pth protein. Cultures were centrifuged and the cells were lysed for $10 \mathrm{~min}$ at $100^{\circ} \mathrm{C}$ in lysis buffer $(50 \mathrm{mM}$ Tris $/ \mathrm{HCl}$, pH 6.8, $100 \mathrm{mM}$ DTT, 2\% SDS, 0.1\% bromophenol blue). Proteins were separated by electrophoresis on $15 \%$ polyacrylamide gels as described by Laemmli (1970). Gels were dried and the radioactivity in individual bands was determined when required using a phosphorimager (Molecular Dynamics). Transfer to nitrocellulose membranes and Western blotting with antibodies (diluted 5000-fold) were performed as described by Sambrook et al. (1989), using ${ }^{125}$ I-labelled protein A (Amersham) to reveal the antibody.

Southern blotting. Following restriction enzyme digestion, DNA fragments were separated by gel electrophoresis in $0.7 \%$ agarose gels. Phage $\lambda$ DNA digested with BstEII and labelled with $\left[\gamma^{32} \mathrm{P}\right] \mathrm{ATP}$ was used as molecular mass marker. A labelled probe for the $p t h$ gene was prepared by PCR amplification of the complete gene followed by labelling with a Ready Prime II labelling kit (Pharmacia) according to the manufacturer's instructions.

\section{RESULTS}

\section{Reversion rate of the thermosensitive pth mutation depends strongly on selection temperature and genetic background}

Previous studies have shown that the apparent frequency of reversion of the $p t h(\mathrm{ts})$ mutation isolated by Atherly \& Menninger (1972) varies widely according to the nonpermissive temperature employed, the plating medium and the strain background (Heurgué-Hamard et al., 1996; V. Heurgué-Hamard \& L. Mora, unpublished observations). Extragenic suppressor mutations such as the tRNA missense suppressor affecting the $g l y W$ gene were selected at temperatures of $8-10{ }^{\circ} \mathrm{C}$ above the threshold for thermosensitive growth, in strains with low apparent rates of reversion, consistent with point mutation rates (Heurgué-Hamard et al., 1996). To obtain more quantitative data on mutation rates under different conditions, we determined the proportion of small independent cultures that acquired no revertants over a period of growth, as described by Luria \& Delbrück (1943). These measurements allowed calculation of the reversion probability per cell per generation. Two $p t h($ ts) strains were studied, VH733 and
VH4, with very different threshold temperatures for thermosensitivity (Table 2). In both cases, however, when the reversion rate was measured at $4{ }^{\circ} \mathrm{C}$ above this threshold, high rates of reversion were observed, in the range $10^{-3}-10^{-5}$. The more thermosensitive strain, $\mathrm{VH} 4$, allowed the study of reversion rates over a range of temperature, and showed a striking decrease in reversion rate to $10^{-8}$ at $42^{\circ} \mathrm{C}$ (Table 2 ).

Several factors may contribute to the differences, due to strain background and the temperature dependence of the reversion rate. The frequency of drop-off may vary between strains and is probably itself a temperaturedependent event (Cruz-Vera et al., 2000). Residual Pth activity may be present in the strains at temperatures that are non-permissive for growth, and the level of such activity in relation to the volume of peptidyl-tRNA drop-off may determine the type of mutational event that restores growth. The level of Pth synthesis or the stability of thermosensitive Pth may vary between strains. Previous work had thrown light on the type of infrequent mutation that can suppress growth thermosensitivity of the mutant $p$ th strain, but the nature of the reversion events observed at a high rate under certain conditions remained unknown. To distinguish between some of the possiblities outlined above we attempted to answer the following question: do the revertants arising at a high rate require residual Pth activity for growth?

\section{Revertants selected under conditions of high reversion rate are due to duplication of the pth gene}

A collection of $p t h(\mathrm{ts})$ revertants was made from a large number of independent cultures of the $p t h(\mathrm{ts})$ strain VH733 under conditions of high reversion rate, conserving one revertant only per culture. To determine whether the $p t h$ gene was still necessary for the growth of these revertants, a strain (JMM35) was constructed in which the chromosomal $p$ th locus is interrupted by a cassette conferring kanamycin resistance, with the requirement for Pth met by a plasmid (pBADPth) carrying a wild-type $p$ th gene expressed from an arabinose-inducible promoter. Each of the thermoresistant revertant strains, either before or after transformation with pBADPth, was transduced with a P1 lysate made on JMM35, selecting for kanamycin resistance in the presence of arabinose. Transductants of the thermosensitive control strain VH733 could only be obtained if the strain carried the plasmid pBADPth prior to transduction, as expected in view of the essential

Table 2. Rates of reversion to thermoresistance of pth(ts) strains

\begin{tabular}{|lccc|}
\hline Strain & $\begin{array}{c}\text { Threshold for } \\
\text { thermosensitivity }\left({ }^{\circ} \mathrm{C}\right)\end{array}$ & $\begin{array}{c}\text { Temp. of reversion } \\
\left({ }^{\circ} \mathrm{C}\right)\end{array}$ & $\begin{array}{c}\text { Reversion rate per cell } \\
\text { per generation }\end{array}$ \\
\hline VH733 & 39 & 43 & $5 \times 10^{-4}$ \\
VH4 & 34 & 42 & $10^{-8}$ \\
VH4 & 34 & 40 & $2 \times 10^{-6}$ \\
VH4 & 34 & 38 & $10^{-5}$ \\
\hline
\end{tabular}


(a)

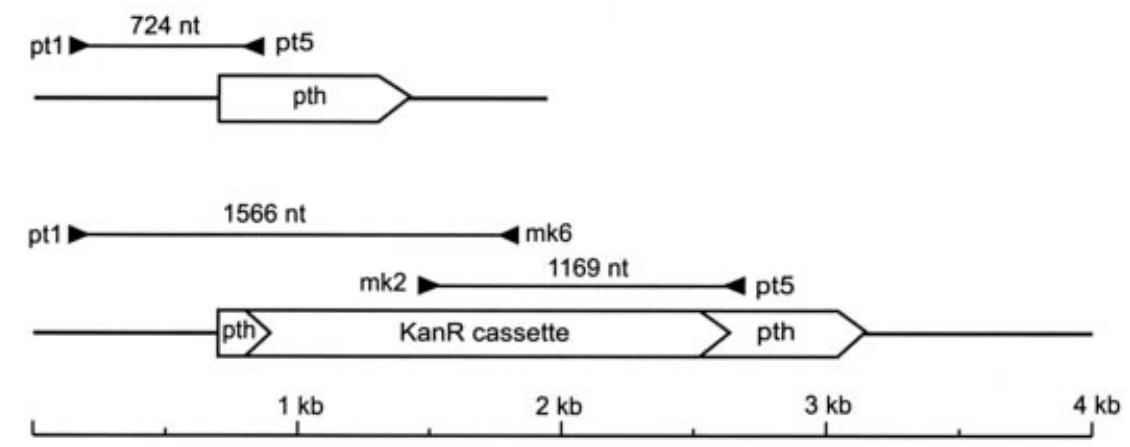

(b)

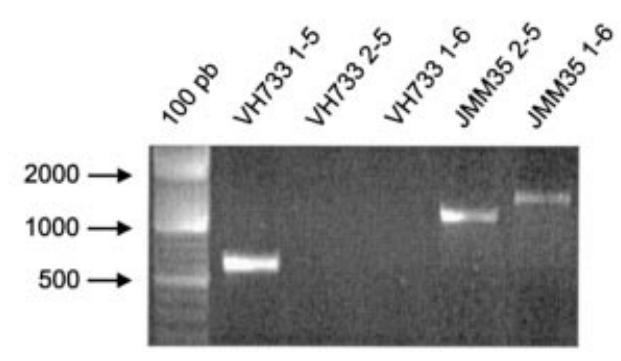

(c)

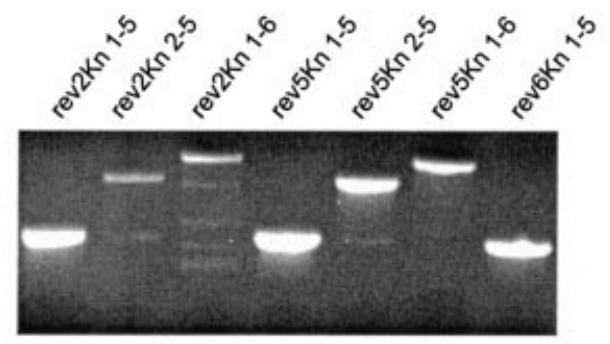

(d)

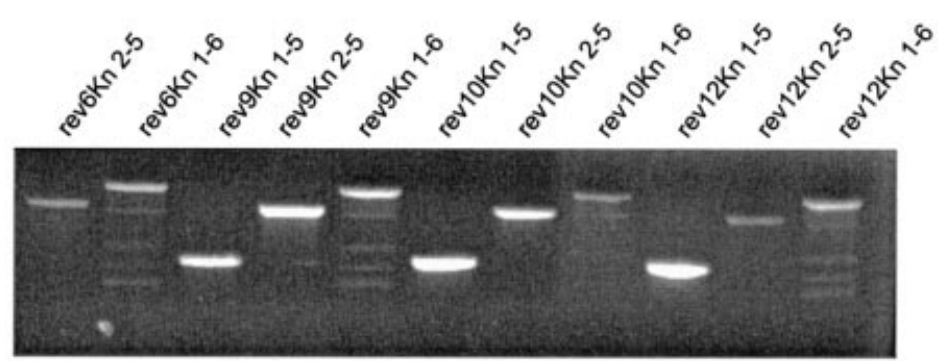

Fig. 1. $P C R$ analysis of DNA from thermoresistant revertants of strain VH733 after transduction of $p$ th::Kn. DNA from thermoresistant revertants of the pth(ts) strain $\mathrm{VH} 733$ obtained at $43{ }^{\circ} \mathrm{C}$, transduced to kanamycin resistance with pth:: $\mathrm{Kn}$, was analysed with PCR primers having sequences present in the parent strain (pt1 and pt5) and in the kanamycin-resistant insert ( $\mathrm{mk} 2$ and $\mathrm{mk} 6$ ). Part (a) shows the positions of the primer pairs and the lengths of the expected PCR products. Part (b) shows the products obtained from amplifications on control strains VH733 [pth(ts)] and JMM35 (pth::Kn, pBAD), with a molecular mass ladder (100 pb); parts (c) and (d) show the products of amplification from six of the thermoresistant revertants after transducion to kanamycin resistance. Lanes are labelled with the numeric part of the primer pairs (thus, $1-6$ is the pair pt1-mk6).

nature of the pth gene. In contrast, each of the thermoresistant revertant strains yielded about 200 kanamycin-resistant transductants whether or not the plasmid was present. Each of the plasmid-free trans- duced revertants became once more thermosensitive, except in one case (numbered rev5). Similar experiments were performed with the more thermosensitive strain $\mathrm{VH} 4$, selecting thermoresistant revertants at $38^{\circ} \mathrm{C}$. In 
this case also the revertants, but not the parent strain, could be transduced to kanamycin resistance with $p t h:: \mathrm{Kn}$.

These observations suggested that thermoresistance had been acquired by duplication of the mutant pth gene. Three types of experiment were performed to test this hypothesis, by PCR amplification of the pth gene, Southern blot analysis, and Western blot analysis using anti-Pth sera. First, PCR amplifications were performed on revertants and transduced revertants. A pair of primers was chosen in the region neighbouring $p t h$ (see Fig. 1a), one upstream of the $p t h$ gene $(p t 1)$ and one within the coding sequence (pt5). In the case of both the transduced and non-transduced revertants, a fragment of the size corresponding to the normal pth gene was amplified, confirming the existence of a non-interrupted locus (Fig. 1b-d). A further specific primer (mk6) was chosen in the middle of the kanamycin-resistance gene introduced into $p$ th which, in combination with pt1, amplified a fragment of the expected length $(1.6 \mathrm{~kb})$ only in the case of the transduced revertants, confirming the presence of the interrupted $p$ th gene (Fig. $1 \mathrm{~b}-\mathrm{d}$ ). Thus, it is clear that the revertants harbour at least two pth loci, one of which is interrupted on transduction with a P1 phage lysate on strain JMM35. The transduced revertants thus showed both the presence of a normal pth gene and a further interrupted copy of the gene (Fig. 1b).

Southern blot analysis was performed with probes specific to $p t h$ and to the kanamycin-resistance cassette on DNA extracted from FTP4993 and the 12 thermoresistant revertants after digestion with $E c o R V$. A single fragment was seen in each case (see Fig. 2b, c), of the expected size, $6.6 \mathrm{~kb}$, as in the case of the VH733 parent strain (Fig. 2b, lane 2) and the Pth wild-type strain Xac (Fig. 2c, lane 2). After the transduction of the revertants with pth::Kn described above, a second fragment appeared in addition to that of $6.6 \mathrm{~kb}$, of the size expected $(11.6 \mathrm{~kb})$ in the case of insertion of the kanamycin-resistance cassette into pth. Analysis of DNA from JMM35 showed no $6.6 \mathrm{~kb}$ fragment, but only the chromosomal inactivated fragment and a $4.8 \mathrm{~kb}$ molecule from the linearized pBADPth plasmid (Fig. 2b, lane 3 ). The identity of the more slowly migrating fragments was confirmed by hybridization first with the anti-Kn probe, followed by partial dehybridization and subsequent hybridization with the anti-pth probe (Fig. $2 b, c)$. No new fragments were visible in the case of the revertants, suggesting that the region of the chromosome containing $p$ th and undergoing duplication is probably larger than the region of about $6 \mathrm{~kb}$ covered by the restriction fragments.

\section{Western blot analysis of Pth from different strains and thermoresistant revertants}

The preceding experiments indicated that Pth is synthesized in the thermoresistant revertants even after inactivation of one chromosomal copy of the $p t h$ gene. To confirm this and look for possible differences in Pth level in the revertants compared to the parent strain, we performed Western blot analysis on cell extracts. The level of Pth in strain VH733 was around the limit of detection in these assays, whereas all revertants showed clearly detectable Pth (Fig. 3). Inactivation of a chromosomal pth copy in the revertants by transduction of $p t h:: \mathrm{Kn}$ reduced the apparent level of Pth to near or below the level of detection except in the case of one revertant (rev5), the single case in which transduction did not restore thermosensitive growth to the revertant. Revertant rev5 may harbour more than two copies of the pth locus, by triplication for example. It is also possible that a duplication has placed the pth gene in a more expressed region, such as near the origin of replication.

The Western blot in Fig. 3 shows the slight difference in migration between wild-type Pth from strain FTP4993 and Pth from strains carrying the $p t h(\mathrm{ts})$ allele (CruzVera et al., 2000). Other experiments showed that the level of wild-type Pth in C600 and FTP4993 strains was closely similar, but insufficient sensitivity of the Western blotting technique precluded any comparison of Pth levels in the $p t h$ (ts) derivatives of these strains. Purified Pth enabled the Western blots to be calibrated, showing that both wild-type strains contain about $1000 \mathrm{~mol}-$ ecules of Pth per cell (not shown). This is comparable to the value of 1300 molecules per cell found recently by Cruz-Vera et al. (2000) and significantly greater than an earlier report of 25 molecules per cell (Dutka et al., 1993).

\section{Reversion rate following shut-off of Pth synthesis}

In the $p t h(\mathrm{ts})$ mutant the capacity of Pth to hydrolyse the peptidyl-tRNA dissociating from the ribosome diminishes with increasing temperature, until a threshold temperature is reached at which Pth activity becomes inadequate to sustain cell growth. This may be due to a reduction in Pth activity with temperature or to an increase in the rate of formation of cytoplasmic peptidyl-tRNA, the observations of Cruz-Vera et al. (2000) suggesting that the latter is of greater importance. In either case, the experiments described above show that the cell is able to counter an inadequate level of Pth activity within a certain range of temperatures above the threshold temperature for growth by multiplication of the number of copies of the mutant $p t h$ gene. This interpretation predicts that if the intracellular level of Pth is reduced to a small fraction of its value in the parent cell, this mechanism will no longer function. We therefore constructed strains in which Pth synthesis is under the control of a tight inducible promoter, and measured reversion frequencies after shut-off of $p t h$ expression.

Preliminary experiments with strain JMM35, in which wild-type Pth is expressed exclusively from the arabinose-inducible pBAD promoter on a low-copynumber plasmid, showed that many hours of growth were necessary after arabinose elimination to dilute the intracellular Pth. A similar result was obtained with the arabinose-inducible $p$ th system recombined with the 
(a)

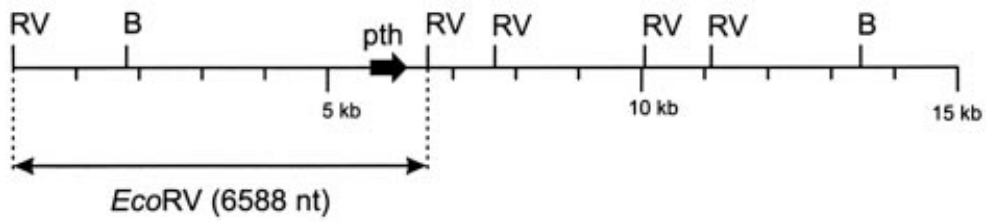

(b)

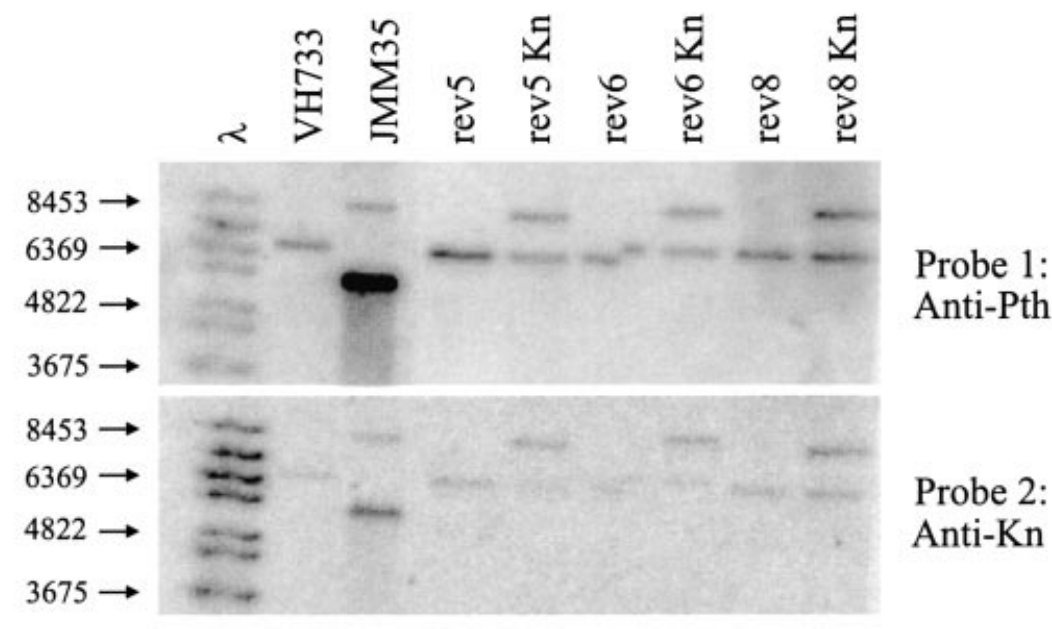

(c)

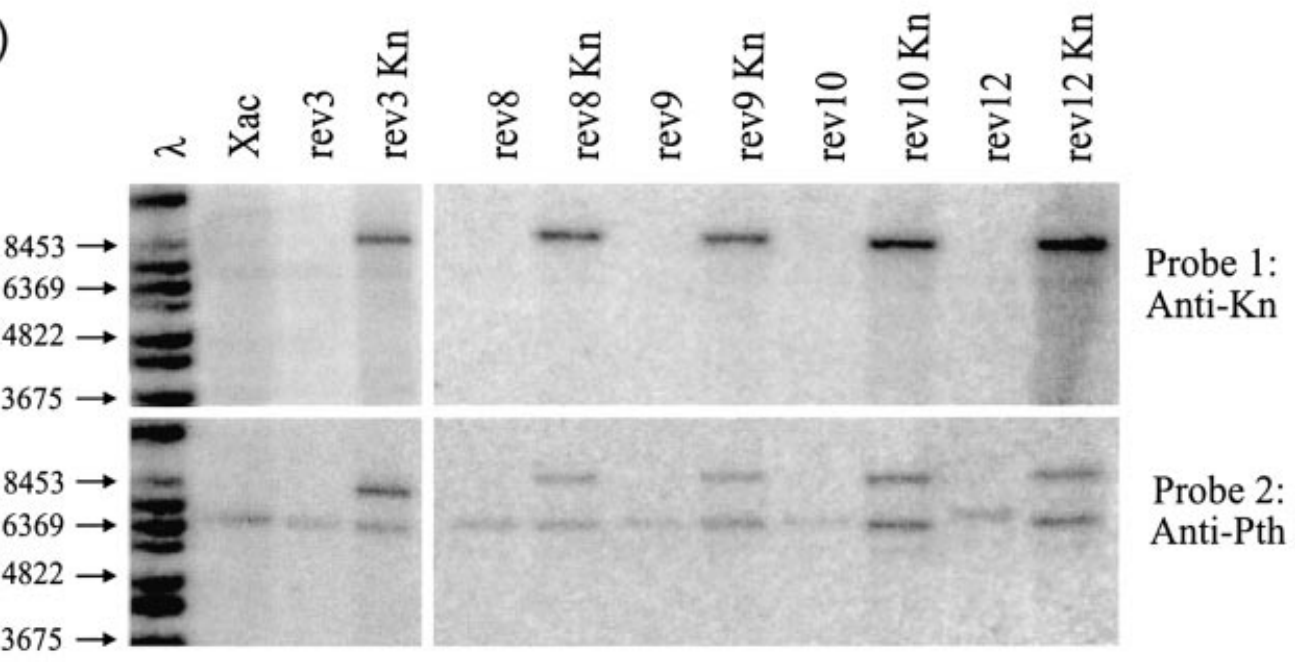

Fig. 2. Southern blot analysis of DNA from thermoresistant revertants of strain VH733 before and after transduction of pth::Kn. Part (a) shows the EcoRV sites close to the pth gene and the expected size of the fragments containing the gene, before insertion of the Kn cassette. DNA from pth wild-type strain Xac, pth(ts) strain VH733, strain JMM35 in which the $p$ th gene is carried on plasmid pBADpth, seven thermoresistant revertants (rev3, 5, 6, 8, 9, 10 and 12) and their pthinterrupted derivatives (rev3 $\mathrm{Kn}$, etc) were digested with EcoRV and subjected to Southern blot analysis. Two ${ }^{32} \mathrm{P}-$ labelled probes were used, specific to the pth gene (amplified between pt1 and pt5; see Fig. 1) and to the Kn cassette used to interrupt the pth locus (mk2 to mk6; Fig. 1). In each case, the two different probes were used successively on the same membrane, partially dehybridized after the first hybridization. Part (b) shows the results of hybridization with DNA from strains VH733, JMM35 and three thermoresistant revertants first hybridized with the pth probe and secondly with the $\mathrm{Kn}$ probe. Part (c) shows a similar analysis of five revertant strains, except that the anti-Kn probe was used before the anti-pth probe. The left track in (b) and (c) indicates marker fragments from phage $\lambda$ DNA digested with BstEll. 


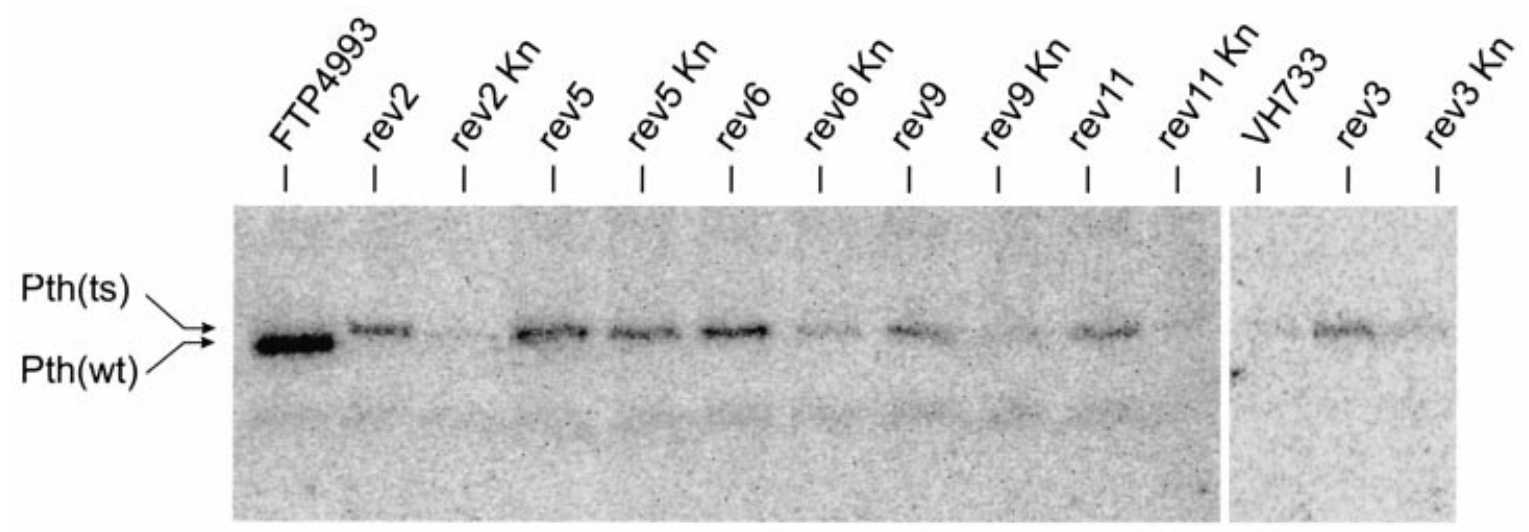

Fig. 3. Western blot analysis of proteins extracted from thermoresistant revertants of a pth(ts) mutant before and after introduction of $p t h:: \mathrm{Kn}$ by transduction. Western blotting with rabbit anti-Pth antibody was performed as described in Methods. Lanes rev2 $\mathrm{Kn}$, rev3 $\mathrm{Kn}$, etc., refer to strains after introduction of $p$ th::Kn by transduction from strain JMM35. The other six revertants characterized were similar to the class including rev2, 3, 6, 9 and 11. The wild-type Pth strain FTP4993 and thermosensitive strain VH733 are included as controls.

chromosome at the maltose operon locus (data not shown). A plasmid was therefore constructed equivalent to $\mathrm{pBADpth}$ but encoding the thermosensitive enzyme, and used to transform FTP4993, in which the chromosomal $p t h$ gene was then inactivated as in JMM35. In the presence of $0.02 \%$ arabinose, the resulting strain is similar in behaviour to a 'chromosomal' $p t h$ (ts) strain. This construction has the advantage that upon elimination of arabinose not only is the synthesis of new Pth molecules arrested, but the short half-life of the thermosensitive Pth mutant results in a rapid decrease in the intracellular concentration of the enzyme. The reversion rate at $43{ }^{\circ} \mathrm{C}$ was studied as described above, and there was a total absence of revertants. We estimate that this corresponds to a reversion rate lower than $3 \times 10^{-9}$.

\section{DISCUSSION}

In certain genetic backgrounds and within a certain range of temperatures that are non-permissive for growth, the thermosensitive $p t h$ mutation reverts to thermoresistance at a high frequency. We have shown here that the mechanism of reversion is one of amplification of the mutant $p t h$ gene. With the exception of one of the revertants studied, the data are consistent with a simple duplication of the gene. This implies that a substantial range of conditions exists in which a modest increase in Pth activity is sufficient to restore cell growth.

At first sight, it might seem surprising that there should be such a critical level of Pth activity required for growth, such that a mere doubling in level of the enzyme at a given temperature should suffice to restore normal or near normal growth. A likely explanation for this is suggested by a consideration of the mechanism of peptidyl-tRNA drop-off from the ribosome. It was shown by Menninger (1978) that different families of peptidyl-tRNAs accumulate at rates differing by as much as 200-fold after shift of a thermosensitive Pth strain to non-permissive temperature. Studies of the kinetics of drop-off of different peptidyl-tRNAs from the cognate codon on the ribosome have failed to show large differences in rate (Dinçbas et al., 1999; Dinçbas-Renqvist et al., 2000; V. Heurgué-Hamard, unpublished data). Likewise, the specificity of Pth towards different peptidyl-tRNA substrates does not show variations sufficient to account for the range of rates of peptidyl-tRNA accumulation (Dinçbas et al., 1999; Dinçbas-Renqvist et al., 2000; V. HeurguéHamard, unpublished data). These observations support the conclusion of Menninger (1978) that a substantial part of peptidyl-tRNA drop-off arises from translational errors; thus, drop-off occurs as a result of a weak semi-cognate codon-anticodon interaction rather than a cognate interaction. In this case, it is readily understandable that conditions under which Pth is rather limiting in the cell, leading to partial starvation for some species of aminoacyl-tRNA, will favour translational errors and increase yet further peptidyltRNA drop-off, imposing a greater substrate load on the available Pth. This may suffice to explain the need for a critical level of the enzyme.

Gene amplification is an extensively documented phenomenon in prokaryotic cells and can extend from simple duplication of a gene to amplification of more than 100-fold (for recent reviews, see Bachellier et al., 1966; Hughes, 1999). Several examples in E. coli and Salmonella typhimurium have been described, such as the duplication at rates of $10^{-5}$ or more per cell per generation of a mutant Gly-tRNA synthetase gene (Folk \& Berg, 1971), amplification of the $\arg F$ region in some Hfr strains of E. coli (Jessop \& Clugston, 1985) and amplification of the lac region (Tlsty et al., 1984). Amplification is generally dependent on repeated sequences within the genome, such as the rrn genes (Anderson \& Roth, 1981), insertion elements (Jessop \& 
Clugston, 1985), REP sequences (Shyamala et al., 1990) or $r$ hs repeated sequence elements (Lin et al., 1984).

Although this has not been conclusively shown, it is probable that all currently identified mechanisms of suppression of the thermoresistance of $p t h(\mathrm{ts})$ depend on continued Pth activity in the cell. It is also clear that with the exception of the duplication mechanism shown here, reversion occurs at low frequency, of the order of $10^{-8}$ per cell per generation or lower, consistent with point-mutation events (Drake, 1969).

Saccharomyces cerevisiae chromosome VIII (Ouzounis et al., 1995) and fragments of mammalian and mouse origin available in public databases reveal the existence of $p$ th genes of the prokaryotic type. Biochemical studies of rabbit reticulocytes, however, have revealed another type of Pth activity, due to a phosphodiesterase which removes the terminal adenosine of tRNA together with the peptide, which appear then to be cleaved in a subsequent step (Gross et al., 1992a, b). Recycling of the tRNA then requires repair of the 3 ' terminus by tRNACCA nucleotidyl transferase activity. It remains to be seen whether either the prokaryotic-type Pth activity or the phosphodiesterase-type Pth activity is essential to cell viability in cells of higher organisms.

\section{ACKNOWLEDGEMENTS}

We thank Valérie Heurgué-Hamard for bacterial strains and plasmids and both her and Miklos de Zamaroczy for helpful comments on the manuscript. This work was supported by the Centre National pour la Recherche Scientifique (UPR9073), Aventis Pharma France (convention de recherche PES0781098), l'Association pour la Recherche sur le Cancer and the Fondation pour la Recherche Medicale.

\section{REFERENCES}

Anderson, P. \& Roth, J. (1981). Spontaneous tandem genetic duplications in Salmonella typhimurium arise by unequal recombination between rRNA (rrn) cistrons. Proc Natl Acad Sci U S A 78, 3113-3117.

Atherly, A. G. \& Menninger, J. R. (1972). Mutant Escherichia coli strain with temperature sensitive peptidyl-transfer RNA hydrolase. Nature 240, 245-246.

Bachellier, S., Gilson, E., Hofnung, M. \& Hill, C. W. (1966). Repeated sequences. In Escherichia coli and Salmonella: Cellular and Molecular Biology. Edited by F. C. Neidhardt and others. Washington, DC: American Society for Microbiology.

Blomfield, I. C., Vaughn, V., Rest, R. F. \& Eisenstein, B. I. (1991). Allelic exchange in Escherichia coli using the Bacillus subtilis $s a c B$ gene and a temperature-sensitive pSC101 replicon. Mol Microbiol 5, 1447-1457.

Coulondre, C. \& Miller, J. H. (1977). Genetic studies of the lac repressor III: additional correlation of mutational sites with specific aminoacid residues. J Mol Biol 117, 525-575.

Cruz-Vera, L. R., Toledo, I., Hernandez-Sanchez, J. \& Guarneros, G. (2000). Molecular basis for the temperature sensitivity of Escherichia coli pth(Ts). J Bacteriol 182, 1523-1528.

De La Vega, F. M., Galindo, J. M., Old, I. G. \& Guarneros, G.
(1996). Microbial genes homologous to the peptidyl-tRNA hydrolase-encoding gene of Escherichia coli. Gene 169, 97-100.

Dinçbas, V., Heurgué-Hamard, V., Buckingham, R. H., Karimi, R. \& Ehrenberg, M. (1999). Shutdown in protein synthesis due to the expression of mini-genes in bacteria. J Mol Biol 291, 745-759.

Dinçbas-Renqvist, V., Engström, Å., Mora, L., Heurgué-Hamard, V., Buckingham, R. H. \& Ehrenberg, M. (2000). A post-translational modification in the GGQ motif of RF2 from E. coli stimulates termination of translation. EMBO J 19, 6900-6907.

Drake, J. W. (1969). Comparative rates of spontaneous mutation. Nature 221, 1132.

Dutka, S., Meinnel, T., Lazennec, C., Mechulam, Y. \& Blanquet, S. (1993). Role of the 1-72 base pair in tRNAs for the activity of Escherichia coli peptidyl-tRNA hydrolase. Nucleic Acids Res 21, 4025-4030.

Folk, W. R. \& Berg, P. (1971). Duplication of the structural gene for glycyl-transfer RNA synthetase in Escherichia coli. J Mol Biol 58, 595-610.

Gross, M., Crow, P. \& White, J. (1992a). The site of hydrolysis by rabbit reticulocyte peptidyl-tRNA hydrolase is the $3^{\prime}$-AMP terminus of susceptible tRNA substrates. J Biol Chem 267, 2080-2086.

Gross, M., Starn, T. K., Rundquist, C., Crow, P., White, J., Olin, A. \& Wagner, T. (1992b). Purification and initial characterization of peptidyl-tRNA hydrolase from rabbit reticulocytes. J Biol Chem 267, 2073-2079.

Guzman, L. M., Belin, D., Carson, M. J. \& Beckwith, J. (1995). Tight regulation, modulation, and high-level expression by vectors containing the arabinose PBAD promoter. J Bacteriol 177, 4121-4130.

Hamilton, C. M., Aldea, M., Washburn, B. K., Babitzke, P. \& Kushner, S. R. (1989). New method for generating deletions and gene replacements in Escherichia coli. J Bacteriol 171, 4617-4622.

Heurgué-Hamard, V., Mora, L., Guarneros, G. \& Buckingham, R. H. (1996). The growth defect in E. coli deficient in peptidyltRNA hydrolase is due to starvation for Lys-tRNA ${ }^{\text {Lys }}$. EMBO J 15, 2826-2833.

Heurgué-Hamard, V., Karimi, R., Mora, L., MacDougall, J., Leboeuf, C., Grentzmann, G., Ehrenberg, M. \& Buckingham, R. H. (1998). Ribosome release factor RF4 and termination factor RF3 are involved in dissociation of peptidyl-tRNA from the ribosome. EMBO J 17, 808-816.

Hughes, D. (1999). The impact of homologous recombination on genome organisation and stability. In Organisation of the Prokaryotic Genome, pp. 109-128. Edited by R. L. Charlebois. Washington, DC: American Society for Microbiology.

Jessop, A. P. \& Clugston, C. (1985). Amplification of the $\arg F$ region in strain HfrP4X of E. coli K-12. Mol Gen Genet 201, 347-350.

Jørgensen, F. \& Kurland, C. G. (1990). Processivity errors of gene expression in Escherichia coli. J Mol Biol 215, 511-521.

Laemmli, U. K. (1970). Cleavage of structural proteins during the assembly of the head of bacteriophage T4. Nature 227, 680-685.

Lin, R. J., Capage, M. \& Hill, C. W. (1984). A repetitive DNA sequence, rhs, responsible for duplications within the Escherichia coli K-12 chromosome. J Mol Biol 177, 1-18.

Luria, S. E. \& Delbrück, M. (1943). Mutations of bacteria from virus sensitivity to virus resistance. Genetics 28, 491-511.

Manley, J. L. (1978). Synthesis and degradation of termination and premature termination fragments of beta-galactosidase in vitro. J Mol Biol 125, 407-432. 
Menez, J., Heurgue-Hamard, V. \& Buckingham, R. H. (2000). Sequestration of specific tRNA species cognate to the last sense codon of an overproduced gratuitous protein. Nucleic Acids Res 28, 4733-4741.

Menninger, J. R. (1978). The accumulation as peptidyl-transfer RNA of isoaccepting transfer RNA families in E. coli with temperature-sensitive peptidyl-transfer RNA hydrolase. $J$ Biol Chem 253, 6808-6813.

Miller, J. H. (1992). A Short Course in Bacterial Genetics. Cold Spring Harbor, NY: Cold Spring Harbor Laboratory.

Ouzounis, C., Bork, P., Casari, G. \& Sander, C. (1995). New protein functions in yeast chromosome VIII. Protein Sci 4, 2424-2428.

Sambrook, J., Fritsch, E. F. \& Maniatis, T. (1989). Molecular Cloning: a Laboratory Manual, 2nd edn Cold Spring Harbor, NY : Cold Spring Harbor Laboratory.
Schmitt, E., Mechulam, Y., Fromant, M., Plateau, P. \& Blanquet, S. (1997). Crystal structure at $1.2 \AA$ resolution and active site mapping of Escherichia coli peptidyl-tRNA hydrolase. EMBO J 16, 4760-4769.

Shyamala, V., Schneider, E. \& Ames, G. F. (1990). Tandem chromosomal duplications: role of REP sequences in the recombination event at the join-point. EMBO J 9, 939-946.

Tlsty, T. D., Albertini, A. M. \& Miller, J. H. (1984). Gene amplification in the lac region of E. coli. Cell 37, 217-224.

Vogel, Z., Zamir, A. \& Elson, D. (1968). On the specificity and stability of an enzyme that hydrolyses $N$-substituted aminoacyltransfer RNAs. Proc Natl Acad Sci U S A 61, 701-707.

Received 2 February 2001; accepted 2 March 2001. 